\title{
Facile Doping in Two-Dimensional Transition Metal Dichalcogenides by UV Light
}

\author{
Ly, Thuc Hue ; Deng, Qingming; Doan, Manh Ha ; Li, Lian-Jong; Zhao, Jiong
}

Published in:

A C S Applied Materials and Interfaces

Link to article, DOI:

10.1021/acsami.8b09797

Publication date:

2018

Document Version

Peer reviewed version

Link back to DTU Orbit

Citation (APA):

Ly, T. H., Deng, Q., Doan, M. H., Li, L-J., \& Zhao, J. (2018). Facile Doping in Two-Dimensional Transition Metal Dichalcogenides by UV Light. A C S Applied Materials and Interfaces, 10(35), 29893-29901.

https://doi.org/10.1021/acsami.8b09797

\section{General rights}

Copyright and moral rights for the publications made accessible in the public portal are retained by the authors and/or other copyright owners and it is a condition of accessing publications that users recognise and abide by the legal requirements associated with these rights.

- Users may download and print one copy of any publication from the public portal for the purpose of private study or research.

- You may not further distribute the material or use it for any profit-making activity or commercial gain

- You may freely distribute the URL identifying the publication in the public portal

If you believe that this document breaches copyright please contact us providing details, and we will remove access to the work immediately and investigate your claim 


\title{
Facile Doping in Two-Dimensional Transition Metal
}

\section{Dichalcogenides by UV Light}

Thuc Hue Ly ${ }^{1,}$, Qingming Deng ${ }^{2,3}$, Manh Ha Doan ${ }^{4,5}$, Lain-Jong $\mathrm{Li}^{6}$, Jiong Zhao ${ }^{7}$

${ }^{l}$ Department of Chemistry and Center of Super-Diamond \& Advanced Films (COSDAF), City University of Hong Kong, Hong Kong, China. ${ }^{2}$ Physics Department, School of Physics and Electronic Electrical Engineering \& and Jiangsu Key Laboratory for Chemistry of LowDimensional Materials, Huaiyin Normal University, Huaian 223300, China. ${ }^{3}$ Department of Energy Conversion and Storage, Technical University of Denmark, Fysikvej, 2800 Kgs. Lyngby, Denmark. ${ }^{4}$ IBS Center for Integrated Nanostructure Physics, Institute for Basic Science, Sungkyunkwan University, Suwon 440-746, Korea. ${ }^{5}$ Department of Energy Science, Sungkyunkwan University, Suwon 440-746, Korea. ${ }^{6}$ Physical Sciences and Engineering, King Abdullah University of Science and Technology, Thuwal 23955-6900, Saudi Arabia. ${ }^{7}$ Department of Applied Physics, The Hong Kong Polytechnic University, Hong Kong, China.

"Corresponding author E-mail: (T. H. Ly) thuchly@cityu.edu.hk

\author{
KEYWORDS \\ two dimensional, transition metal dichalcogenides, doping, atomic defects, atomic force \\ microscopy, transmission electron microscopy




\section{ABSTRACT}

Two-dimensional (2D) materials have been emerging as potential candidates for the next generation materials in various technology fields. The performance of the devices based on these 2D materials depends on their intrinsic band structures as well as the extrinsic (doping) effects such as surrounding chemicals and environmental oxygen/moisture, which strongly determines their Fermi energy level. Herein, we report the UV treatments on the 2D transition metal dichalcogenides (TMdCs), to controllably dope the samples without damaging the crystal structures or quenching the luminescence properties. More surprisingly, both $\mathrm{n}$ type and $\mathrm{p}$ type doping can be achieved depending on the initial status of the sample and the UV treatment conditions. The doping mechanisms were elaborated on the atomic scale with transmission electron microscopy and ab-initio calculations. The facile doping by UV light has potential to be integrated with photolithography processes, aiming for the large scale integrated device/circuits design and fabrications.

\section{INTRODUCTION}

Two-dimensional (2D)-layered materials have attracted great attentions for their extraordinary properties, e.g. the electron confinement without interlayer interaction, high mechanical flexibility as well as optical transparency. ${ }^{1}$ Particularly, graphene has zero bandgap which made difficulties for the applications in industry. ${ }^{2}$ On the contrary, $2 \mathrm{D}$ transition metal dichalcogenides (TMdCs), with direct gaps from 1.0 to $2.5 \mathrm{eV},{ }^{1}$ are more suitable for various electronic or optoelectronic applications. Doping is a common process for tailoring the properties of semiconductors. So far a few doping methods for the 2D-TMdCs have been reported, e.g. heat treatment approaches such as chemical vapor deposition (CVD) were enabled for stoichiometric control $^{3}$ or $\mathrm{Nb}$ doping in $\mathrm{MoS}_{2},{ }^{4}$ while wet chemistry methods could be applied for atomic doping in TMdCs as well. ${ }^{5}$ However, most of existed doping methods involve either thermal or chemical processes, which are difficult to control or integrated with other processes during large scale fabrications. Some other doping methods, such as plasma treatment approaches ${ }^{6-8}$ were proposed for the carrier control in $\mathrm{MoS}_{2}$ monolayers as well, and they have indeed paved the way for the 2D TMDs towards scalable industrial fabrications. 
On the other hand, currently the detailed structures of defects ${ }^{9-16}$ and oxygen passivation effects on the defects ${ }^{17,18}$ in $2 \mathrm{D}$ TMdCs remain under hot debate. Several previous works ${ }^{19-23}$ have shown that the enhancement of photoluminescence (PL) intensity achieved by thermal annealing or weak plasma irradiation is closely related to strong oxygen bonding at the defect sites of $1 \mathrm{~L}-\mathrm{MoS}_{2}{ }^{19,22,23}$ and the quenching of PL intensity by strong plasma irradiation is associated with the lattice distortion or creation of $\mathrm{MoO}_{3}$ defects. ${ }^{19-21}$ The gradual oxidation of TMdCs are prevalent for these atomic-thick layer when exposed to air, even under ambient conditions. ${ }^{24-27}$ The environmental effects play key roles on the optical and electronic properties of monolayer TMdCs. Therefore, it is imperative to have a systematic study with careful engineering on all control parameters and rationalize the influences on associated structural/property changes, further, for understanding the underlying mechanisms of oxidation.

Distinct to the previous approaches, in this work we apply a facile and industrial friendly method to dope the 2D-TMdCs in both types (n/p). During the initial stage of UV light exposure with humidity, which has been used to oxidize and observe the grain boundaries of graphene ${ }^{27}$ and $\mathrm{TMdCs}^{28}$ the monolayer TMdCs were successfully doped and modified without compromising the structures. According to the summary in Supporting Table S1, the initial stage can last few min, depending on the specific conditions. By careful characterizations with the PL spectroscopy, Raman spectroscopy, XPS, TEM/STEM, density functional theory (DFT) simulation and C-AFM, it is clearly revealed that under a controlled UV irradiation dose and humid level, the chemical doping process can adjust the carriers in bands of 2D-TMdCs, without lattice periodicity/band structure perturbation or sample damage.

In specific, our PL and Raman spectroscopy measurements unveiled the different carrier and lattice vibration behaviors according to the UV irradiation time, humidity level. The PL intensity of exfoliated monolayer (1L) $\mathrm{MoS}_{2}$ gradually increased with significant blue shift of A exciton peak, distinct to the PL quenching with red shift of A exciton peak observed in CVD grown 1L $\mathrm{MoS}_{2}$, indicating different doping effects in these two kinds of samples. Meanwhile, a significant peak shift of the $\mathrm{E}_{2 \mathrm{~g}}^{1}$ and $\mathrm{A}_{1 \mathrm{~g}}$ modes in the Raman spectra for all samples has been observed. The origin of the different doping effects which can be attributed to different defective structures after treatment was unraveled by the high resolution TEM/STEM imaging. Finally, the increased electrical current level on the CVD grown $\mathrm{MoS}_{2}$ samples in the conducting atomic force 
microscopy (C-AFM) results also confirmed the doping. Our work manifests the capability of controlled UV light treatment for tuning the electrical and optical properties of the 2D TMdCs. This process has great advantage over other doping methods and potential for industry use, in particular for the compatibility with current lithography techniques.

\section{RESULTS AND DISCUSSIONS}

The fabrication, preparation and characterization specifications of the 2D TMdCs can be referred to the METHODS. As the first example, the $1 \mathrm{~L} \mathrm{MoS}_{2}$ flakes were exfoliated on 300nm $\mathrm{SiO}_{2} / \mathrm{Si}$ substrate using Scotch-tape method (pristine samples in Supporting information Figure S1) and applied treatment with UV irradiation at humidity level of 60-65\% (Figure 1a-h). Several bright spots/holes were observed in Figure 1a in the monolayer portion, whereas the thicker portion does not have any noticeable effect. The thickness of monolayer was $\sim 0.8 \mathrm{~nm}$ verified by AFM (Figure 1b,e). The roughness in monolayer part was less than $0.2 \mathrm{~nm}$. The bright spots in Figure 1a were correlated with the dark spots in Figure 1b, showing the formation of nanoholes after UV irradiation under $60 \%$ humidity. The edge height increased to $4 \mathrm{~nm}$, indicating the highly active edges were preferably oxidized as in our previous reports. ${ }^{28}$ The formation of holes and edge oxidation are related to the initial oxidation and etching of $1 \mathrm{~L} \mathrm{MoS}_{2}$ under UV treatment. It should be noted that except for several discrete spots, the samples under UV exposure for $6 \mathrm{~min}$ and $60 \%$ humidity kept globally uniform and exhibited well-preserved uniformity (Figure 1e) as well as crystallinity in $1 \mathrm{~L} \mathrm{MoS}_{2}$, double-confirmed by our TEM characterizations shown later.

In contrast, increasing of 5\% humidity gave different results. For $65 \%$ humidity and 6 min UV exposure samples, there were continuous bright lines and bright spots appeared in the AFM images (Figure 1d,f). Even in the $>1 \mathrm{~L}$ portions there were some changes in morphology. Line patterns emerged with height $\sim 0.8 \mathrm{~nm}$ and width $\sim 50 \mathrm{~nm}$, roughness was also increased to $\sim 3$ $\mathrm{nm}$ (Figure 1f), which probably comes from the functionalization groups after oxidation of $\mathrm{MoS}_{2}{ }^{19-22}$. Meanwhile, stress corrosion cracking (SCC) ${ }^{29}$ was also initiated, with cracking depth $0.8 \mathrm{~nm}$ (1L thickness) and width $\sim 50 \mathrm{~nm}$.

To investigate the physical properties after UV treatment, we performed spectroscopic analysis by Raman and photoluminescence (PL) on the initially oxidized samples. Figure 1g,h exhibit the results on the $6 \mathrm{~min}$ UV and $60 \%$ humidity treatment samples. Firstly, the two 
characteristic peaks from Raman spectra for original monolayer $\mathrm{MoS}_{2}$ at $384.2 \mathrm{~cm}^{-1}$, assigned to $\mathrm{E}_{2 \mathrm{~g}}^{1}$ mode, and $401.5 \mathrm{~cm}^{-1}$, assigned to $\mathrm{A}_{1 \mathrm{~g}}$ mode, were observed. ${ }^{8}$ After UV oxidation for $6 \mathrm{~min}$, the $E^{1}{ }_{2 \mathrm{~g}}$ peak was slightly shifted to $383.6 \mathrm{~cm}^{-1}$ and $\mathrm{A}_{1 \mathrm{~g}}$ peak was shifted to $402.5 \mathrm{~cm}^{-1}$. In the PL results, The A exciton peak (excited by a $532 \mathrm{~nm}$ laser) of $1 \mathrm{~L} \mathrm{MoS}_{2}$ near $1.82 \mathrm{eV}$ was blueshifted with enhanced peak intensity. Accordingly, the intensity of the B exciton peak near 805 $\mathrm{nm}$ was significantly reduced without appreciable peak shifting. These results are all in consistent with the $p$ type doping effects ${ }^{5,30}$ for the $2 \mathrm{D} \mathrm{MoS}_{2}$. Based on the Raman peak shift we obtained, the actual free electron density reduction ( $p$ doping) was estimated to be $2 \times 10^{12} \mathrm{~cm}^{-2}$. 30 The increased total PL intensity is well contrasted to the behavior after other thermal and plasma treatments, ${ }^{31}$ whereas the PL intensity is normally reduced significantly.

In line with our AFM morphology results, the increased PL intensity after UV exposure confirmed that the crystal structure of $2 \mathrm{D} \mathrm{MoS}_{2}$ has not been degraded, otherwise the prevalent dangling bonds will serve as non-recombination centers and reduce the PL intensity. A exciton peak is combined with the negative trion peak $(\sim 1.84 \mathrm{eV})$ and exciton peak $(\sim 1.88 \mathrm{eV}) .^{5}$ Compared to the as-exfoliated $\mathrm{MoS}_{2}$ samples, the blue shift of A exciton peak and peak intensity enhancement at around $1.88 \mathrm{eV}$ after UV oxidation can be attributed to the transition of negative trion recombination to direct exciton recombination, ${ }^{4}$ in congruent with the decrease of free electrons due to $\mathrm{p}$ type doping. The $65 \%$ humidity and 6 min UV treated samples (Figure 1d,f) have much lower PL intensity than the $60 \%$ humidity and 6 min UV treated samples (Supporting Figure S1), exhibiting they have been severely oxidized and damaged.

The mechanical exfoliated samples usually contain less atomic defects compared to the CVD grown samples, especially the atomic vacancies in $2 \mathrm{D}$ TMdCs. ${ }^{14}$ As the second example, CVDgrown monolayer $\mathrm{MoS}_{2}$ on sapphire ${ }^{32}$ was tested without transferring substrates to reduce unexpected artifacts caused by the transfer process. The high quality pristine samples before UV exposure were shown in Supporting Figure S2. While after UV irradiation under 65\% humid level for $6 \mathrm{~min}$, Figure 2a showed the topographic AFM image demonstrating the uniformity of the triangle shape $1 \mathrm{~L} \mathrm{MoS}_{2}$ (thickness $\sim 0.8 \mathrm{~nm}$, roughness $\sim 0.1 \mathrm{~nm}$ ). Only several brighter lines caused by the SCC cracks due to initial oxidation and large strain mismatch between the flake and sapphire substrate ${ }^{29,33}$. It is also reasonable that the CVD grown samples were cracked under a lower humidity level than mechanical exfoliated samples with same exposure time, because the 
CVD samples intrinsically possess more atomic defects or vacancies than the mechanical exfoliated samples.

To further understand the oxidation effects, we prepared samples with different UV exposure times, while the humidity level maintained at $65 \%$ and $60 \%$. The series of AFM results for $65 \%$ humidity were presented in Supporting Figure S3, and the height profiles confirmed the uniform morphologies except some cracks or oxidized edges/boundaries starting to emerge at 4 min while larger particles were seen after 6 min. The series of Raman and PL spectra for $65 \%$ humidity was shown in Figure 2b-e, and the respective Raman and PL (mapping) data were presented in Supporting Figure S4-S7. It can be seen that the Raman intensity kept spatially uniform in the initial stage but the total intensity was increased after UV irradiation until 4 min and then start to rapidly decrease from 6 min (Figure 2d). The enhanced Raman intensity could be ascribed to plasmonic effects from the oxidation process. ${ }^{4}$ Figure $2 \mathrm{f}$ showed the peak position evolution of $E_{2 g}^{1}$ and $A_{1 g}$. Both $E_{2 g}^{1}$ and $A_{1 g}$ peaks were shifted to lower wavenumber with the increasing UV exposure time (0-4 min), and then shift back higher wavenumber for longer exposure time $(>4$ min). The initial and subsequent shifting of $\mathrm{E}_{2 \mathrm{~g}}^{1}$ peak can be well explained by the strain effects $^{34-36}$ in response to the UV irradiation. With more atomic defects/vacancies created, the tensile stress across the sample was increased, thereby the sample started to have SCC cracks and the tensile stress was eventually released. On the other hand, the $\mathrm{A}_{1 \mathrm{~g}}$ peak position is more closely related to the doping concentrations. ${ }^{30}$ The corresponding PL spectra also presented the crossover of $\mathrm{n} / \mathrm{p}$ doping by A exciton peaks at around 2 3 min UV exposure (Supporting Figure $\mathrm{S} 4 \mathrm{~b}$ ). The crossover is earlier than the $\mathrm{A}_{1 \mathrm{~g}}$ peak in Raman spectra (crossover at $4 \mathrm{~min}$ ), which is probably due to the effect of in-plane strain mixed with doping in the PL results, say, the tensile strains cause similar effect as p doping for PL. The above results indicate in the initial stage of UV treatment, $n$ type doping tends to occur first in the CVD grown samples followed by $p$ type doping, in contrast to the direct $\mathrm{p}$ type doping by UV in the mechanical exfoliated samples.

The initial $\mathrm{n}$ type doping effect for CVD samples was confirmed by the PL measurements. The A exciton peak was red-shifted (Figure 2c) for 5 min UV irradiated sample. This red-shift A exciton peak can be attributed to $n$ type doping ${ }^{5}$ or tensile straining, ${ }^{30}$ however the 5 min UV sample was already SCC cracked (Figure 2a), therefore most of the tensile stress is already released, precluding the tensile strain effect. Nevertheless, $\mathrm{n}$ type doping is supposed to dominate 
the initial stage during the UV oxidation process in the CVD grown samples. The maximum electron density ( $\mathrm{n}$ doping) estimated by Raman shift was increased by $6 \times 10^{12} \mathrm{~cm}^{-2}$ after UV doping. Finally after around 6 min by the UV treatment, the $1 \mathrm{~L} \mathrm{MoS}_{2}$ started to be damaged (Supporting Figure S8), which significantly reduced the Raman and PL intensities.

Hence we have demonstrated the morphology and optical responses of the monolayer $\mathrm{MoS}_{2}$ samples, both mechanical exfoliated and CVD grown, can be controllably modified by the UV light and rich humid exposure. Conditions including the thickness of sample, particularly monolayer, as well as the exposure time and humidity level will result in distinct reaction rates. Importantly, during the initial stage (e.g. $<6$ min under $60 \%$ humidity or $<4$ min under $65 \%$ humidity, using current UV exposure intensity and dose, see METHODS) of treatment, doping effect on the monolayer $\mathrm{MoS}_{2}$ were clearly observed while uniform morphologies can be preserved. As the monolayers intrinsically have higher chemical reactivity than thicker flakes due to fluctuations in morphology, higher surface ratios and higher defect concentrations, they suit such mild UV oxidative treatment for doping. Because 5 min UV exposure is under the threshold between the mild doping (initial stage) and the stronger oxidation/damage, in the following we mainly use the 5 min UV exposure sample to demonstrate the doping effect.

The $\mathrm{MoS}_{2}$ layer was further studied by the X-ray photoelectron spectroscopy (XPS) for elemental analysis. Figure 3 showed the XPS spectra of the Mo-3d and S-2s of pristine and post UV treatment $1 \mathrm{~L} \mathrm{MoS}_{2}$. After 5 min UV irradiation, the S-2s peak was suddenly reduced (Figure 3 upper panel), shown in Table 1, by around 50\%. Note the sapphire substrates also contribute in the $\mathrm{O}$ signals. The oxygen content after $5 \mathrm{~min}$ UV treatment was significantly increased (Table 1). The accuracy of $\mathrm{Mo} / \mathrm{S}$ ratio determined by XPS may be disturbed by X ray beam damage, however it clearly exhibited the trend of replacement of S by O during the UV treatment. In addition, the individual peak of $\mathrm{Mo} \mathrm{d}^{6+}$ which related to oxidation was significantly increased after UV, and the core level of S-2p and Mo-3d peaks was red shifted simultaneously by around $1.5 \mathrm{eV}$ due to UV exposure and chemical doping (Figure 3).

By using high resolution TEM and STEM techniques, we have been able to observe the local atomic structures of the UV treated TMdCs (Figure 4a-f). The annular dark field (STEM-ADF) image (Figure 4a) showed the CVD grown $1 \mathrm{~L} \mathrm{MoS}_{2}$ have perfect crystal lattice even after 5 min UV irradiation under $60 \%$ humidity, and the mechanical exfoliation samples have similar results. 
Although there are some atomic vacancies in S sublattice (Figure 4d), the Mo sublattice is nonaffected. The oxygen substitution into the $\mathrm{S}$ atomic positions cannot be clearly resolved by ADF because the $\mathrm{O}$ and $\mathrm{S}$ are both light elements and quite close in $\mathrm{Z}$ contrast, however the localized EELS spectrum over defective zones (Figure 4b,c) confirmed the trace of oxygen. While in pristine $\mathrm{MoS}_{2}$ monolayer samples without UV treatment, it is hardly to find trace of oxygen in the EELS spectra for local areas (Supporting Figure S9). Therefore, the O substitution of S or O atom/OH functional group (caused by the photochemical dissociation of $\mathrm{H}_{2} \mathrm{O}$ ) attaching to $\mathrm{S}$ vacancies will deplete the original $\mathrm{n}$ type carriers (electrons) in conduction band caused by $\mathrm{S}$ vacancies, leading to significant p-doping effect, which is in consistent with the PL and Raman spectroscopy results (Figure 1).

In addition, in CVD grown samples, by using ADF imaging we frequently observed sulphur vacancies (Figure 4d) and additional Mo atoms replacing S atoms (Figure 4e,f), which can enhance n-type doping by excess electron (by additional Mo atoms) injection into the conduction band. As more atomic defects and S vacancies were found in as-prepared CVD grown samples, the creation of extra sulphur vacancies can be easier than less defective exfoliated $\mathrm{MoS}_{2}$, because the $\mathrm{S} 2$ vacancies in $\mathrm{MoS}_{2}$ are energy favorable. ${ }^{16}$ On anther hand, the Mo atoms in the original lattice are more active upon oxidation conditions. Additional Mo atoms may also come from the $\mathrm{MoO}_{\mathrm{x}}$ sources used for CVD process. The n-doping effect can be saturated due to exhaustion of $\mathrm{S}$ vacancies or Mo sources, which is followed by $\mathrm{p}$ doping effect similar to the mechanical exfoliated samples. Elongated UV exposure time $(>8 \mathrm{~min})$ or levitated humidity level on the samples will quickly damage or even destroy the samples.

To confirm the electronic structures of the O/Mo doped $1 \mathrm{~L}-\mathrm{MoS}_{2}$, we carried out DFT calculations (Figure 5). The single $\mathrm{S}_{\mathrm{O}}$ defect and $\mathrm{S}_{\mathrm{Mo}}$ doping defects which were observed by TEM experiments are mainly investigated. The structure and local density of states (LDOS) of monolayer $\mathrm{MoS}_{2}$ shows a band gap of ca. $1.8 \mathrm{eV}$ in Figure 5b, which are consistent with previous DFT calculations ${ }^{37,38}$. The gap is mainly derived from $4 \mathrm{~d}$ orbits of Mo atoms. The symmetry of LDOS is kept as shown as in Figure $5 \mathrm{c}$ by substituting one $\mathrm{S}$ atom with $\mathrm{O}$. The $2 \mathrm{p}$ orbitals of $\mathrm{O}$ locate far from the Fermi level $(<-2.4 \mathrm{eV})$ indicating slightly effect of the chemical properties by substitution $\mathrm{S}$ with $\mathrm{O}$. Therefore, the $\mathrm{O}$ doping will deplete the $\mathrm{n}$ doping levels caused by original S vacancies. For Mo doping, the impurity states distributed around Fermi 
level are mainly contributed from $4 \mathrm{~d}$ orbits of Mo as dopants in Figure 5d, and the dopant Mo 4d defect orbits (in pink, Figure 5d) also locate at the conduction band edges, thereby enhancing ndoping. In brief, the TEM/STEM studies on the post UV samples and DFT calculations have explained the origin of the distinct $\mathrm{n} / \mathrm{p}$ doping effects on the atomic scale.

The electrical properties of the 2D TMdCs after doping were studied by the conductive atomic force microscopy (C-AFM) measurements. The $\mathrm{SiO}_{2}$ layer of $300 \mathrm{~nm} \mathrm{SiO}_{2} / \mathrm{Si}$ substrate was etched away instantly in buffer etching oxide (BOE) solution before the CVD grown $\mathrm{MoS}_{2}$ transferred on the new substrate. The idea here is to use the $\mathrm{MoS}_{2}$ layer as the protection layer for the Si substrate from re-oxidation after etching away the surface oxide. Si was used as the counter electrode for the C-AFM measurements. The C-AFM can provide local I-V responses in correspondence to the topographic information obtained by AFM. The AFM topographic image of the $1 \mathrm{~L} \mathrm{MoS}_{2}$ (thickness $\sim 0.8 \mathrm{~nm}$ ) after UV treatment are shown in Figure 6a. Accordingly, Figure $6 \mathrm{~b}$ showed the current mapping at a bias voltage of $1 \mathrm{~V}$ and contact force of $-1 \mathrm{nN}$. Note there is no much contrast in current level between $\mathrm{MoS}_{2}$ and Si substrate. It can be seen that after UV irradiation for $5 \mathrm{~min}$, brighter spots emerged in the topography image as well as the C-AFM images. The height profiles in Figure 6c,d clearly showed the morphology change of $\mathrm{MoS}_{2}$ after UV irradiation. The peak marked by green arrow in Figure 6c highlighted points with high level of current/conductivity as in Figure 6d, indicating the synchronized increase of current (C-AFM) and height (AFM) in $\mathrm{MoS}_{2}$ due to cracking and severe oxidation. Except for these, the red arrows in Figure 6d also highlighted the high current spots which locate in the uniform/flat areas (no corresponding peaks in height profile in Figure 6c). They can be attributed to the Mo substitution, leading to the enhanced n-doping and increased density of carriers (electrons). The measured current level at the brighter spots in Figure 6d increased approximately by one order of magnitude, from $0.08 \mathrm{~A}$ in pristine samples to $0.8 \mathrm{~A}$ in UV exposed samples, implying the carrier (electron) density increased by one order of magnitude in $\mathrm{MoS}_{2}$. With the initial room temperature carrier (electron) density on order of $10^{12} \mathrm{~cm}^{-2}$ in CVD MoS 2 monolayers, ${ }^{39,40}$, the $\mathrm{n}$ doping concentration after UV exposure can reach around $1 \times 10^{13} \mathrm{~cm}^{-2}$ based on our C-AFM results, which is comparable with the previous results (increase by $5 \times 10^{12} \mathrm{~cm}^{-2}$ ) by optical measurements. The slight difference between them may come from the local characteristics of the C-AFM experiment and the averaging effect in optical measurements. 
In general, the above doping approach by UV irradiation on $2 \mathrm{D} \mathrm{MoS}_{2}$ can be extended to other $\mathrm{TMdC}$ materials such as $\mathrm{WSe}_{2}$ (Supporting Figure S10). The reaction kinetics directly depend on the humidity level and UV exposure time, and the photochemistry of the doping process can be understood as follows. The dissociation of $\mathrm{H}_{2} \mathrm{O}$ molecules are at first triggered by the direct UV absorption. The wavelength of the UV light $(185 \mathrm{~nm})$ we applied has satisfied the energy requirement $(<239 \mathrm{~nm})$ of the $\mathrm{H}_{2} \mathrm{O}$ molecule dissociations. ${ }^{41}$ The full photochemical reactions have been listed in METHODS section. The chalcogenide vacancies in the as prepared samples serve as the anchoring sites for the $-\mathrm{O}$ or $-\mathrm{OH}$ functional groups, which will induce $\mathrm{p}$ type doping. If oxidation processes involve more chalcogenide vacancies such as in CVD grown samples, the encapsulated transition metal atoms become more active and some dissociated metal atoms will act as the n-type dopants. Subsequently, the enhanced oxidization level will turn n-doping into p-doping by oxygen atom substitution similar to mechanical exfoliated samples, after certain amount of UV light and humidity exposure. Eventually, uncontrolled oxidation by UV will cause sample damage and severely deteriorate all the physical properties.

We have summarized the doping results depending on different $\mathrm{MoS}_{2}$ samples, UV doses and humidity levels in supporting Table S1, as a guideline for the doping process and conditions. It is also noted the ideal humid level (process window) for doping in $\mathrm{MoS}_{2}$ should be around $60 \% \sim 65 \%$. Lower or higher humidity will cause too slow or too fast doping reactions which become less controllable. The humidity level is critical to doping due to the introduction of $\mathrm{OH}^{*}$ radicals, meanwhile the effect of $\mathrm{O}_{3}$ on $\mathrm{MoS}_{2}$ is mainly oxidation (with structure damage) rather than doping. Thus under lower humid condition the doping is slower, while more $\mathrm{O}_{3}$ remained in the chamber which can cause faster oxidation (damage) in the sample. The exfoliated $\mathrm{MoS}_{2}$ may have less possibility to react with $\mathrm{O}_{3}$ because they have less defects, therefore the oxidation (damage) is slower. The competition between oxidation (by $\mathrm{O}_{3}$ ) and doping (by $\mathrm{OH}^{*}$ ) exist in the UV process.

In conclusion, we have systematically studied the doping effect of UV irradiation on monolayer TMdCs under humid environment. Controlled $\mathrm{n}$ type or $\mathrm{p}$ type doping is feasible in the initial stage, which can be readily applied for electrical/optical device design and fabrications. By the characterizations using SEM, AFM, TEM, Raman and photoluminescence spectroscopy combined with DFT simulations, we directly compared CVD-grown $\mathrm{MoS}_{2}$ to the mechanical 
exfoliated $\mathrm{MoS}_{2}$ after UV exposure. The PL and Raman spectrum enhancement as well as the electrical conductivity enhancement by C-AFM in the UV treated samples have been observed. This UV approach is facile and compatible with lithography techniques used for industry. It requests only conventional lithography UV dose and ambient humid condition, expected to be cost-effective. In fabrications, the exposure to humid may preclude some processes which require moisture isolation. Meanwhile, the stress in the sample needs to be controlled to prevent SCC cracking. However, this approach still has great potential for the scalable and flexible doping in 2D-TMdCs.

\section{METHODS.}

\section{Sample Preparation.}

Molybdenum dioxide growth on sapphire. The $\mathrm{MoO}_{3}$ powder $(0.3 \mathrm{~g})$ was placed in a ceramic boat located in the heating zone center of the furnace. The S powders were placed in a separate ceramic boat at the upper stream side maintained at $150^{\circ} \mathrm{C}$ during the reaction. The sapphire substrate for growing $\mathrm{MoS}_{2}$ was located at the downstream side, where the $\mathrm{S}$ and $\mathrm{MoO}_{3}$ vapors were brought to the targeting sapphire substrate by an Ar carrier gas (70 sccm, chamber pressure $=40$ Torr). The center heating zone was heated to $635^{\circ} \mathrm{C}$ with a ramping rate $15^{\circ} \mathrm{C} / \mathrm{min}$. After reaching $635^{\circ} \mathrm{C}$, the heating zone was kept for 30 minutes and the furnace was then naturally cooled down to room temperature.

Preparation of exfoliated $1 \mathrm{~L}-\mathrm{MoS}_{2}$ on $\mathrm{Si} / \mathrm{SiO}_{2}$ substrate. $\mathrm{MoS}_{2}$ flake was purchased from 2DMaterials Company, $\mathrm{MoS}_{2}$ was prepared on $300 \mathrm{~nm} \mathrm{SiO}_{2} / \mathrm{Si}$ substrate using conventional mechanical exfoliation methods using the semiconductor transfer tape (USI Blue Adhesive Plastic Film).

Ultraviolet Oxidization of $\mathrm{MoS}_{2}$. $\mathrm{MoS}_{2}$ was placed into a chamber equipped with a low-pressure Hg lamp (LH-arc, Lichtzen, with an output of $20 \mathrm{~mW} \mathrm{~cm}^{-2}$ ), with the majority of emitted light at a wavelength of $254 \mathrm{~nm}$ and approximately $10 \%$ of emitted light at a wavelength of $185 \mathrm{~nm}$. Humidity was introduced into chamber by connecting it to a humidifier. The humidity level in the chamber was monitored using a hydro-thermometer (accuracy of $\pm 3 \%$ ), where the chamber 
was continuously ventilated to maintain a constant pressure. After reaching the required humidity level, the humidifier was disconnected from the chamber. $\mathrm{MoS}_{2}$ was then irradiated under UV light for different times (2, 4, 6, 8 and $10 \mathrm{~min}$ ). Radicals are generated under UV and humidity conditions as follows:

$$
\begin{aligned}
& \mathrm{O}_{2} \rightarrow \mathrm{O}_{3} \\
& \mathrm{O}_{3}+\mathrm{H}_{2} \mathrm{O} \rightarrow \mathrm{O}_{2}+\mathrm{H}_{2} \mathrm{O}_{2} \\
& 2 \mathrm{O}_{3}+\mathrm{H}_{2} \mathrm{O}_{2} \rightarrow 2 \mathrm{OH}^{*}+3 \mathrm{O}_{2} \\
& \mathrm{H}_{2} \mathrm{O} \rightarrow \mathrm{H}^{*}+\mathrm{OH}^{*}
\end{aligned}
$$

Sample Characterization. Raman and PL spectroscopy (NT-MDT) was performed with an excitation energy of $2.33 \mathrm{eV}(532 \mathrm{~nm})$, AFM (SPA400, SEIKO), SEM (FESEM, JSM7000F, JEOL), and TEM (JEM ARM 200F, JEOL Ltd.) were used to characterize the sample. We describe the details of each characterization as below.

Optical microscopy. Optical microscopy (100x magnification, Olympus, Japan, numerical aperture $=0.9$ ) was used to obtain images of the surface morphologies of samples.

Raman and PL spectroscopy. Confocal Raman, PL mapping (NT-MDT, $532 \mathrm{~nm}$ wavelength, NTEGRA Spectra PNL, x100 lens, 0.7 N.A.) was performed using a laser (532 nm) with 30 $\mu \mathrm{W}$ power. The scanned image was obtained at $128 \times 128$ pixels with a grating of $600 \mathrm{~g} / \mathrm{mm}$ to yield a spectral resolution of $<0.1 \mathrm{~cm}^{-1}$ for confocal Raman mapping and with a grating of 1800 $\mathrm{g} / \mathrm{mm}$ to yield a spatial resolution of $200 \mathrm{~nm}$ for confocal PL mapping. The accumulation time for each spectrum was one second for image scanning and 30 seconds for a single spectrum. An area filter was used to extract the Raman spectrum map with an integration of $\mathrm{E}_{2 \mathrm{~g}}^{1}$ peak (368 to $\left.386 \mathrm{~cm}^{-1}\right)$, and PL intensity map (625 to $\left.775 \mathrm{~nm}\right)$.

Atomic force microscopy (AFM). AFM images were obtained using a SPA400 system (SEIKO, Japan) in tapping mode. A NSC14-type silicon tip (MikroMasch, Estonia) with an approximately $10 \mathrm{~nm}$ tip radius was used. In general, the force constant and resonant frequencies of the tips were approximately $5 \mathrm{~N} / \mathrm{m}$ and $160 \mathrm{kHz}$, respectively. 
Conductive atomic force microscopy (C-AFM) measurements. A Au tip with an approximately $10 \mathrm{~nm}$ tip radius was used. The force constant and resonant frequencies of the tips were approximately $1.6 \mathrm{~N} / \mathrm{m}$ and $28 \mathrm{kHz}$, respectively C-AFM study was done monolayer $\mathrm{MoS}_{2}$ transferred on pre-etched Si substrate. IV measurements from $-1 \mathrm{~V}$ to $1 \mathrm{~V}$ were done with $\mathrm{C}$ AFM.

Scanning electron microscopy (SEM) and energy dispersive spectroscopy (EDS). Fieldemission scanning electron microscopy (FESEM) (JSM7000F, Jeol, Japan) was used to examine the surface morphology of samples at different accelerating voltages to obtain a high level of contrast at different magnifications. An X-Max Silicon Drift Detector in a FESEM-JSM7600F system was used for EDS mapping for $10 \mathrm{~min}$. An accelerating voltage of $3 \mathrm{keV}$ was used to obtain sufficiently pronounced signals while retaining sensitivity to the sample surface.

X-ray photoelectron spectroscopy (XPS). XPS was performed using an Al Ka X-ray source (XPS, ESCA2000, VG Microtech). Mo3d, S2s, O1s peak data were collected to analyze the extent of oxidation of monolayer $\mathrm{MoS}_{2}$. X-ray photoelectron spectroscopy (XPS) (QUANTUM 2000, Physical electronics, USA) was performed using focused monochromatic Al Ka radiation $(1486.6 \mathrm{eV})$.

TEM and DF-TEM. TEM imaging was conducted using a JEM ARM 200F machine operated at $80 \mathrm{kV}$, which did not cause any apparent damage to the sample. Acquisition time for dark field (DF) images was one second using the smallest objective lens aperture. The HRTEM imaging acquisition time was $1 \mathrm{~s}$.

ADF-STEM. Annular dark field (ADF)-STEM imaging was conducted using a probe aberration corrected JEM ARM200F, operated at $80 \mathrm{kV}$. High angle annular dark field (HAADF) images were aquired using a $20 \mathrm{mrad}$ convergence angle. Because the low-voltage electron beam was below the damage threshold energy, the pristine $\mathrm{MoS}_{2}$ lattice remains stable and defect-free with regular scans. Images presented in the main text were acquired with the medium-angle annular dark field detector with angle between $50 \mathrm{mrad}$ to $180 \mathrm{mrad}$ and acquisition times of $1 \mu \mathrm{s}$ per pixel. 
EELS. EELS spectrometer (Enfinium, Gatan) working under $80 \mathrm{kV}$ accelerating voltage was applied. The beam current during the experiments were ca. $120 \mathrm{pA}$. The spectrometer was $0.5 \mathrm{eV}$ per channel dispersion with exposure time $0.1 \mathrm{~s}$ per spectrum.

DFT calculations. Spin-polarized density function theory (DFT) calculations are carried out using the Vienna ab initio simulation package (VASP) program package ${ }^{42,43}$ within the projector augmented wave (PAW) pseudo-potentials (PAW). The exchange-correlation functions are described with the generalized gradient approximation (GGA) in the form of the Perdew, Burke, and Ernzernhof (PBE) functional. The kinetic energy cuto $\square$ for the plane-wave basis set is chosen as $500 \mathrm{eV}$. The distance of vacuum layer is set to be more than $20 \AA$, which is sufficient large to avoid interlayer interactions. The electronic SCF tolerance is set to $10^{-5} \mathrm{eV}$. Fully relaxed geometries and lattice constant are obtained by optimizing all atomic positions until the Hellmann-Feynman forces are less than $0.02 \mathrm{eV} / \AA$. The k-points samplings are $4 \times 4 \times 1$ in the Brillouin zone for structural optimizations, while $8 \times 8 \times 1$ are used for the local density of states (LDOS) calculations.

\section{Figures and Figure Legends}



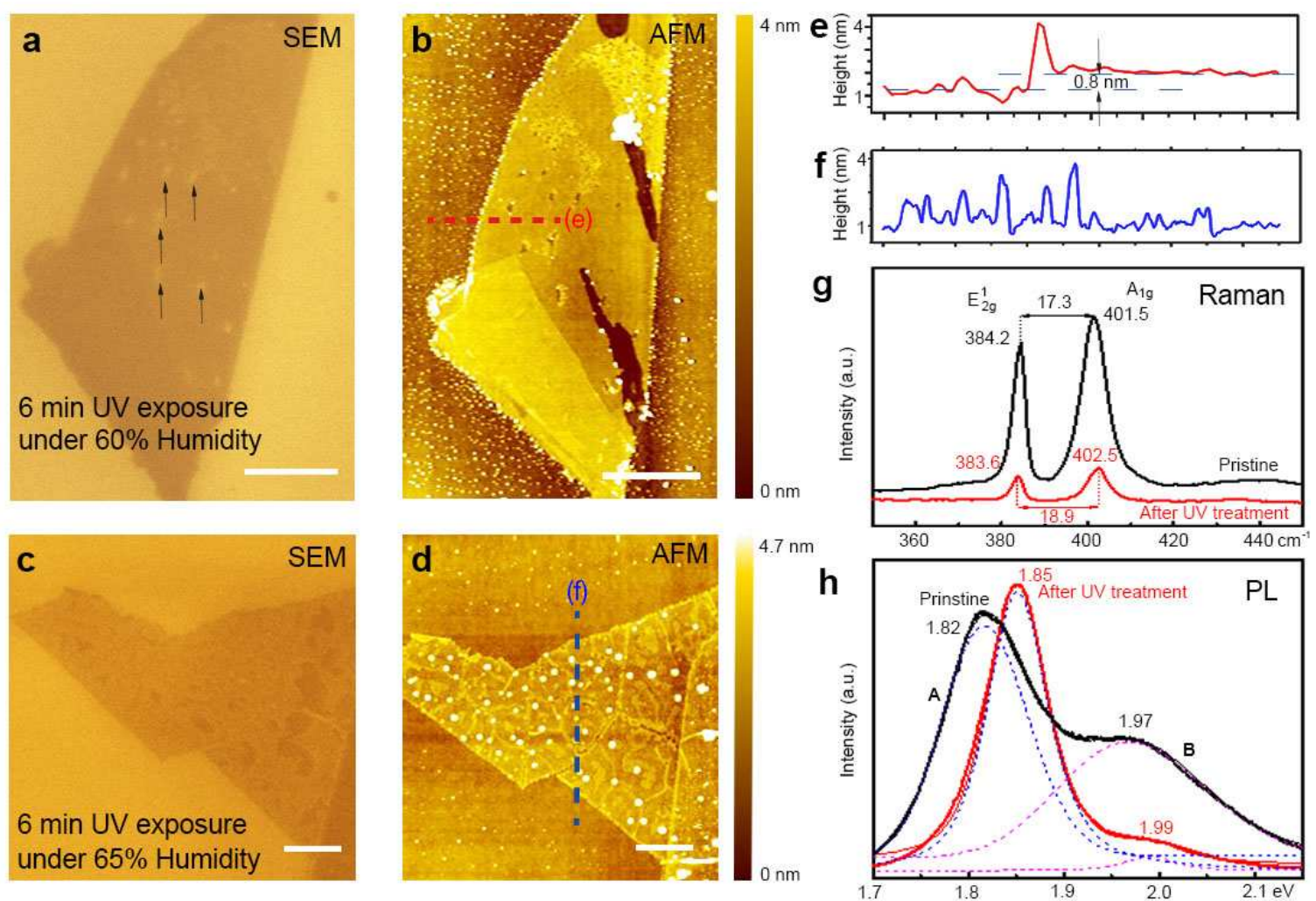

Figure 1. Analysis of UV treatment effect on mechanical exfoliated $\mathrm{MoS}_{2}$. a,c, SEM and b,d, AFM topographic images for the mechanical exfoliated $1 \mathrm{~L} \mathrm{MoS}_{2}$ after 6 min UV treatment under $60 \%$ humidity and $65 \%$ humidity. The black arrows in a marked the nanoholes after UV exposure. Scale bars, a,b, $2 \mu \mathrm{m}, \mathbf{c}, \mathbf{d}, 1 \mu \mathrm{m}$. e,f, Height profiles corresponding to dashed lines in $\mathbf{b , d}, \mathbf{g}$, Raman and $\mathbf{h}$, PL spectra for the pristine and post-UV treatment samples. 

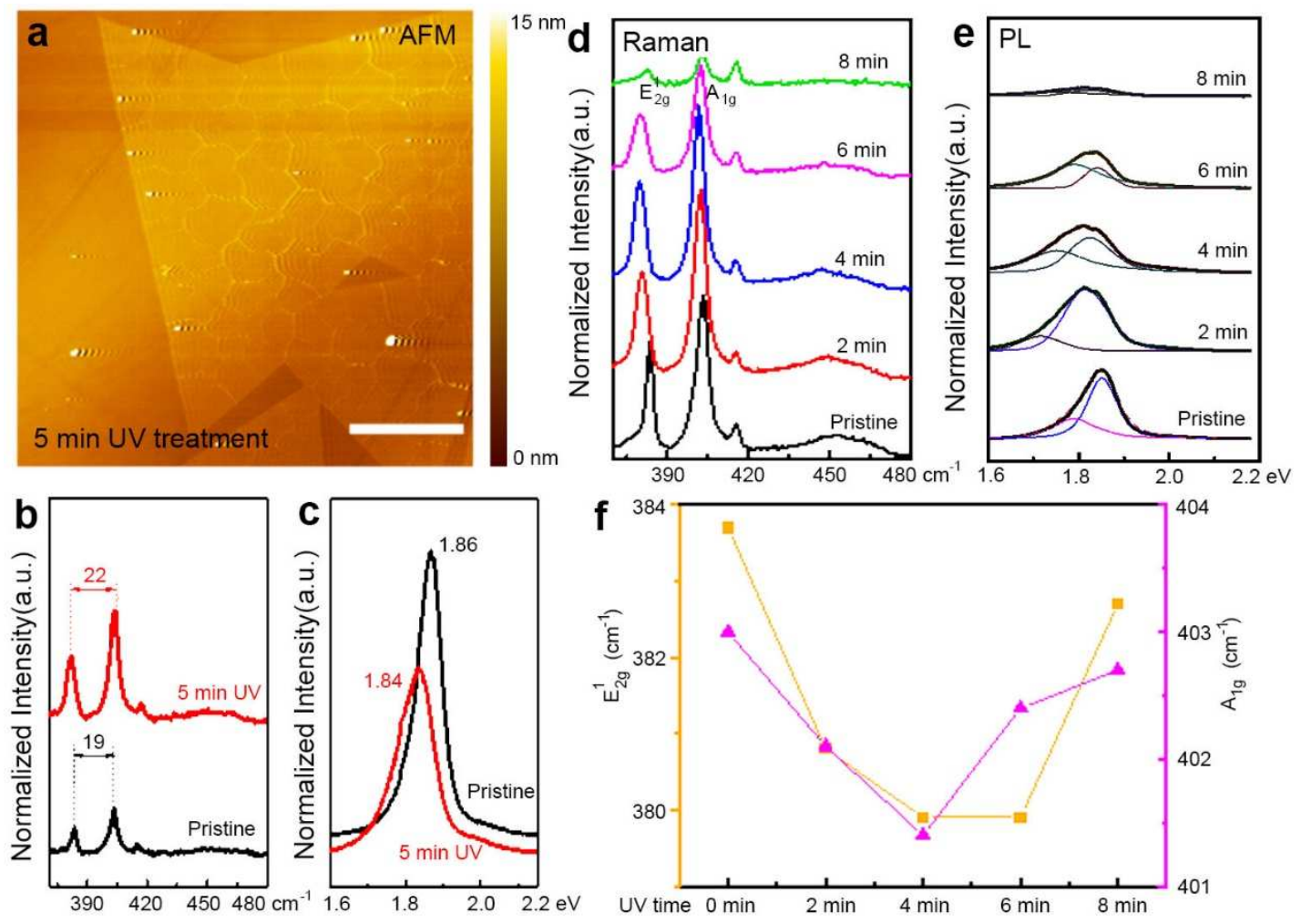

Figure 2. Analysis of CVD grown $1 \mathrm{~L} \mathrm{MoS}$ pre and post UV treatment. a, AFM topographic image for the $1 \mathrm{~L} \mathrm{MoS}_{2}$ after 5 min UV treatment under $65 \%$ humidity. Scale bar, $4 \mu \mathrm{m}$. b,c, Raman and PL analysis of the pristine and 5 min UV treatment sample under $65 \%$ humidity. d,e, Raman and PL analysis of the series of $1 \mathrm{~L} \mathrm{MoS}_{2}$ samples by different UV treatment times under $65 \%$ humidity. $\mathbf{f}$, Time evolution of Raman peaks by UV treatment corresponding to $\mathbf{d}$. 
Figure 3. Chemical components of CVD-MoS ${ }_{2}$ grown on sapphire before (lower panel) and after $5 \mathrm{~min}$ UV treatment under $65 \%$ humidity (upper panel) by XPS. 

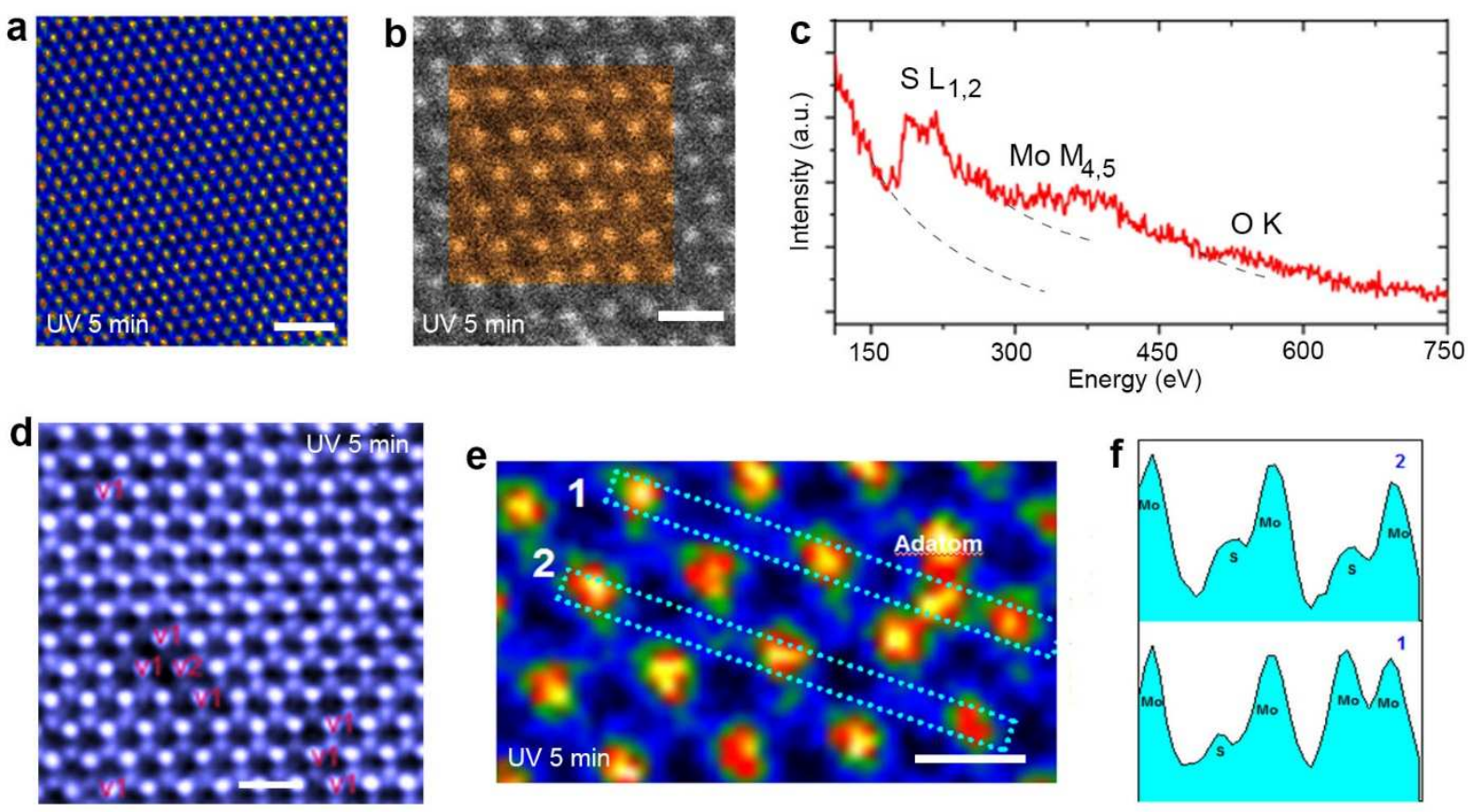

Figure 4. The TEM characterizations of post UV exposure $\mathrm{MoS}_{2}$ samples. False colors are applied for clarity. a, STEM-ADF image for the 5 min UV treatment under $60 \%$ humidity $1 \mathrm{~L}$ $\mathrm{MoS}_{2}$, scale bar $1 \mathrm{~nm}$. b, STEM-ADF image for the $1 \mathrm{~L} \mathrm{MoS}_{2}$ under same condition as a, scale bar 500 pm. c, EELS spectra acquired in the area highlighted in b. d, Filtered ADF image for S vacancy area in $1 \mathrm{~L} \mathrm{MoS}_{2}$ after 5 min UV exposure. Scalr bar, 500 pm. e, Magnified ADF image for the Mo doping area in $1 \mathrm{~L} \mathrm{MoS}_{2}$ after $5 \mathrm{~min}$ UV exposure, scale bar, $300 \mathrm{pm}$. f, Intensity profiles of two dashed lines in e. 
a

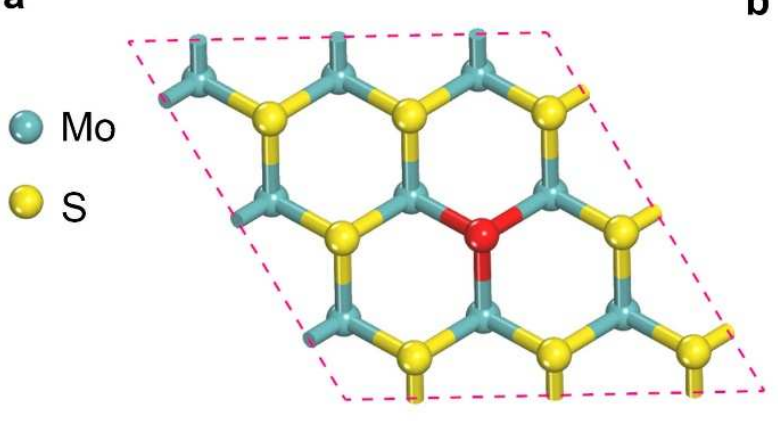

C

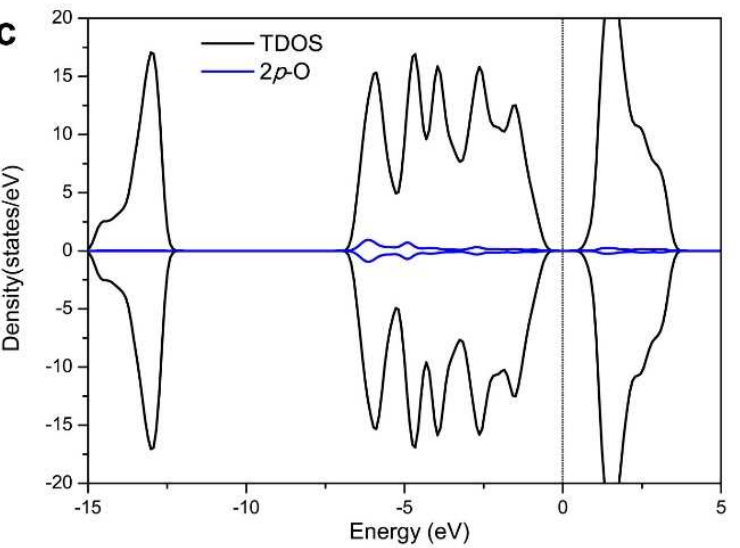

b

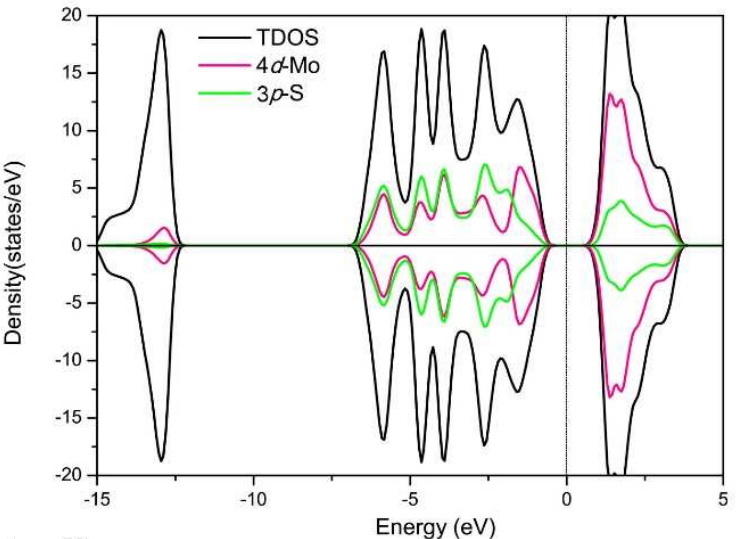

d

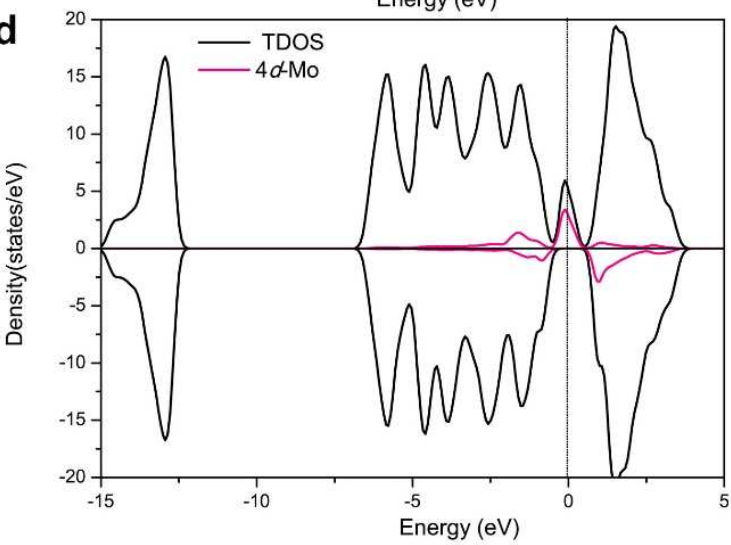

Figure 5. Optimized structure of $\mathrm{MoS}_{2}$ with targeting doping site labelled in red. a, The atomic structures of single atomic doping by $\mathrm{O}$ or Mo substitution for $\mathrm{S}$ in $\mathrm{MoS}_{2}$ (red dopant atom, $\mathrm{O}$ or Mo). Spin-polarized local density of states (LDOS) of $\mathbf{b}$, pristine $\mathrm{MoS}_{2}$, $\mathbf{c}, \mathrm{O}$ doped $\mathrm{MoS}_{2}$, d, Mo doped $\mathrm{MoS}_{2}$ projected on $4 d$ (pink lines) orbitals of Mo as well as $p$ orbitals of $\mathrm{S}$ and $\mathrm{O}$ (green and blue lines), and the total DOS (TDOS) are black lines. The Fermi level is set to zero. 

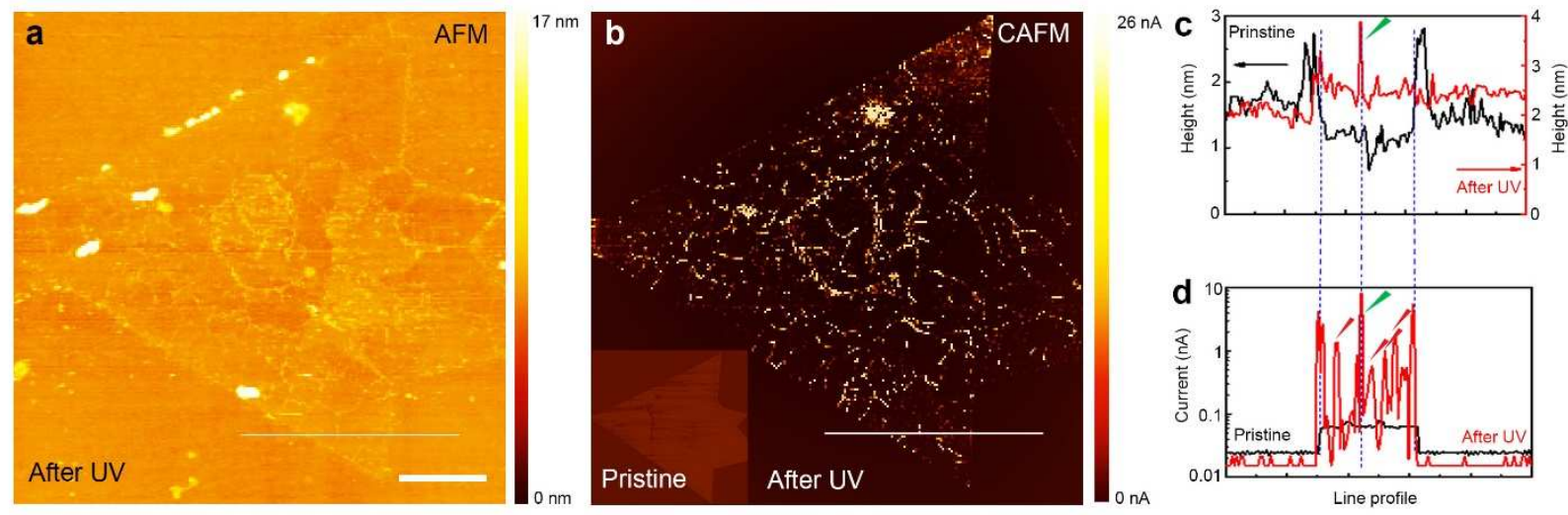

Figure 6. Morphology and conductivity mapping on $\mathrm{UV}$-treated $\mathrm{MoS}_{2}$ on $\mathrm{SiO}_{2} / \mathrm{Si}$ substrate using C-AFM. a, Topography images of CVD grown $\mathrm{MoS}_{2}$ after $5 \mathrm{~min}$ UV treatment, scale bar $4 \mu \mathrm{m}$. b, The C-AFM current mapping for the same position as in a. c,d, The line profiles correspond to the thin white lines in $\mathbf{a}$ and $\mathbf{b}$, and the pristine samples.

Table 1. The detailed data extracted from deconvolution of Mo3d, S2p, and O1s peaks in XPS. Atomic weight calibrated to Mo\%.

$\begin{array}{lll} & \text { Pristine } & 5 \text { min UV } \\ \text { (Mo3d) Mo } & 1.00 & 1.00 \\ \text { (S2p) S } & 2.24 & 1.10 \\ \text { (O1s) O } & 12.80 & 14.06\end{array}$




\section{ASSOCIATED CONTENT \\ Supporting Information.}

Supporting information includes Figure S1 to S11 and Table S1. This material is available free of charge via the internet at http://pubs.acs.org.

\section{AUTHOR INFORMATION}

\section{Corresponding Author}

*E-mail address: thuchly@cityu.edu.hk

Note

The authors declare no competing financial interest.

\section{ACKNOWLEDGMENTS}

This work was supported by grants from City University of Hong Kong (Project No. 7200551, 9610387), the Hong Kong Polytechnic University Grant (No. 1-ZE8C), National Science Foundation of China (21703076), the People Programme (Marie Curie Actions) of the European Union's Seventh Framework Programme (FP7/2007-2013) under REA grant agreement no. 609405 (COFUNDPostdocDTU), Natural Science Foundation of Jiangsu Province of China (BK20170466). 


\section{REFERENCES}

(1) Zhang, H. Ultrathin Two-Dimensional Nanomaterials. ACS Nano 2015, 9, 9451-9469.

(2) Phaedon, A.; Christos, D. Graphene: Synthesis and Applications. Mater. Today 2012, 15, 86-96.

(3) Kim, I. S.; Sangwan, V. K.; Jariwala, D.; Wood, J. D.; Park, S.; Chen, K.-S.; Shi, F.; RuizZepeda, F.; Ponce, A.; Jose-Yacaman, M.; Dravid, V. P., Marks, T. J.; Hersam, M. C.; Lauhon, L. J. Influence of Stoichiometry on the Optical and Electrical Properties of Chemical Vapor Deposition Derived $\mathrm{MoS}_{2}$. ACS Nano 2014, 8, 10551-10558.

(4) Laskar, M. R.; Nath, D. N.; Ma, L.; Il Lee, E. W.; Lee, C. H.; Kent, T.; Yang, Z.; Mishra, R.; Roldan, M. A.; Idrobo, J. C.; Pantelides, S. T.; Pennycook, S. J.; Myers, R. C.; Wu, Y.; Rajan, S. P-type Doping of $\mathrm{MoS}_{2}$ Thin Films Using Nb. Appl. Phys. Lett. 2014, 104, 092104.

(5) Mouri, S.; Miyauchi, Y.; Matsuda, K. Tunable Photoluminescence of Monolayer $\mathrm{MoS}_{2}$ via Chemical Doping. Nano Lett. 2013, 13, 5944-5948

(6) Wi, S.; Kim, H.; Chen, M.; Nam, H.; Guo, L. J.; Meyhofer, E.; Liang, X. Enhancement of Photovoltaic Response in Multilayer $\mathrm{MoS}_{2}$ Induced by Plasma Doping. ACS Nano, 2014, $8,5270-5281$.

(7) Chen, M.; Nam, H.; Wi, S.; Ji, L.; Ren, X.; Bian, L., Lu, S.; Liang, X. Stable Few-Layer $\mathrm{MoS}_{2}$ Rectifying Diodes Formed by Flasma-Assisted Doping. Appl. Phys. Lett. 2013, 103, 142110 .

(8) Yim, S.; Sim, D. M.; Park, W. I.; Choi, M. J.; Choi, J.; Jeon, J.; Kim, K. H.; Jung, Y. S. Surface-Shielding Nanostructures Derived from Self-Assembled Block Copolymers Enable Reliable Plasma Doping for Few-Layer Transition Metal Dichalcogenides. Adv. Funct. Mater. 2016, 26(31), 5631-5640.

(9) Zhang, S., Wang, C. G., Li, M. Y., Huang, D., Li, L. J., Ji, W., Wu, S. Defect Structure of Localized Excitons in a WSe 2 Monolayer. Phys. Rev. Lett. 2017, 119, 046101.

(10) Hong, J.; Hu, Z.; Probert, M.; Li, K.; Lv, D.; Yang, X.; Gu, L.; Mao, N.; Feng, Q.; Xie, L.; Jin, Z.; Wu, D.; Zhang, Z.; Jin, C.; Ji, W.; Zhang, X.; Yuan, J.; Zhang, Z. Exploring Atomic Defects in Molybdenum Disulphide Monolayers. Nat. Commun. 2015, 6, 6293.

(11) Zhou, W.; Zou, X.; Najmaei, S.; Liu, Z.; Shi, Y.; Kong, J., Luo, J.; Ajayan, P. M.; Yakobson, B. I.; Idrobo, J.-C. Intrinsic Structural Defects in Monolayer Molybdenum Disulfide. Nano Lett. 2013, 13, 2615-2622. 
(12) Liu, Z.; Suenaga, K.; Wang, Z.; Shi, Z.; Okunishi, E.; Iijima, S. Identification of Active Atomic Defects in a Monolayered Tungsten Disulphide Nanoribbon. Nat. Commun. 2011, 2, 213 .

(13) Komsa, H. P.; Kurasch, S.; Lehtinen, O.; Kaiser, U.,; Krasheninnikov, A. V. From Point to Extended Defects in Two-Dimensional $\mathrm{MoS}_{2}$ : Evolution of Atomic Structure under Electron Irradiation. Phys. Rev. B 2013, 88, 035301.

(14) Robertson, A. W.; Lin, Y. C.; Wang, S.; Sawada, H.; Allen, C. S.; Chen, Q.; Lee, S.; Lee, G.-D.; Lee, J.; Han, S.; Yoon, E.; Kirkland, A. I.; Kim, H.; Suenaga, K.; Warner, J. H. Atomic Structure and Spectroscopy of Single Metal (Cr, V) Substitutional Dopants in Monolayer $\mathrm{MoS}_{2}$. ACS Nano 2016, 10, 10227-10236.

(15) Lin, Y. C.; Björkman, T.; Komsa, H. P.; Teng, P. Y.; Yeh, C. H.; Huang, F. S.; Li, K.-H.; Jadczak, J.; Huang, Y.-S.; Chiu, P.-W.; Krasheninnikov, A. V.; Suenaga, K. Three-Fold Rotational Defects in Two-Dimensional Transition Metal Dichalcogenides. Nat. Commun. 2015, 6, 6736 .

(16) Zhao, J.; Nam, H.; Ly, T. H.; Yun, S. J.; Kim, S.; Cho, S.; Yang, H.; Lee, Y. H. Chain Vacancies in 2D Crystals. Small, 2017, 13, 1601930.

(17) Park, W.; Park, J.; Jang, J.; Lee, H.; Jeong, H.; Cho, K.; Hong, S.; Lee, T. Oxygen Environmental and Passivation Effects on Molybdenum Disulfide Field Effect Transistors. Nanotechnology 2013, 24, 095202.

(18) Gogoi, P. K.; Hu, Z.; Wang, Q.; Carvalho, A.; Schmidt, D.; Yin, X.; Chang, Y.-H.; Li, L.J.; Sow, C. H.; Neto, A. H. C.; Breese, M. B. H.; Rusydi, A.; Wee, A. T. S. Oxygen Passivation Mediated Tunability of Trion and Excitons in $\mathrm{MoS}_{2}$. Phys. Rev. Lett. 2017, $119,077402$.

(19) Tongay, S.; Suh, J.; Ataca, C.; Fan, W.; Luce, A.; Kang, J. S.;Liu, J.; Ko. C.; Raghunathanan, R.; Zhou, J.; Ogletree, F.; Li, J.; Grossman, J. C.; Wu, J. Defects Activated Photoluminescence in Two-Dimensional Semiconductors: Interplay between Bound, Charged, and Free Excitons. Scientific Reports 2013, 3, 2657.

(20) Nan, H.; Wang, Z.; Wang, W.; Liang, Z.; Lu, Y.; Chen, Q.; He, D.; Tan, P.; Miao, F.; Wang, X.; Wang, J.; Ni, Z. Strong Photoluminescence Enhancement of $\mathrm{MoS}_{2}$ through Defect Engineering and Oxygen Bonding. ACS Nano 2014, 8, 5738-5745.

(21) Oh, H. M.; Han, G. H.; Kim, H.; Bae, J. J.; Jeong, M. S.; Lee, Y. H. Photochemical Reaction in Monolayer $\mathrm{MoS}_{2}$ via Correlated Photoluminescence, Raman Spectroscopy, and Atomic Force Microscopy. ACS Nano 2016, 10, 5230-5236.

(22) Zhang, X.; Jia, F.; Yang, B.; Song, S. Oxidation of Molybdenum Disulfide Sheet in Water under in Situ Atomic Force Microscopy Observation. J. Phys. Chem. C 2017, 121, 99389943. 
(23) Kim, S.; Choi, M. S.; Qu, D.; Ra, C. H.; Liu, X.; Kim, M.; Song, Y. J.; Yoo, W. J. Effects of Plasma Treatment on Surface Properties of Ultrathin Layered $\mathrm{MoS}_{2}$. 2 D Materials 2016, 3, 035002 .

(24) Lin, Y.; Ling, X.; Yu, L.; Huang, S.; Hsu, A. L.; Lee, Y.-H.; Kong, J.; Dresselhaus, M. S.; Palacios, T. Dielectric Screening of Excitons and Trions in Single-Layer MoS 2 . Nano Lett. 2014, 14, 5569-5576.

(25) Mao, N. N.; Chen, Y.; Liu, D.; Zhang, J.; Xie, L. Solvatochromic Effect on the Photoluminescence of $\mathrm{MoS}_{2}$ Monolayers. Small 2013, 8, 1312-1315.

(26) Chow, W. L.; Luo, X.; Quek, S. Q.; Tay, B K. Evolution of Raman Scattering and Electronic Structure of Ultrathin Molybdenum Disulfide by Oxygen Chemisorption. Adv. Electro. Mater. 2015, 1, 1400037.

(27) Ly, T. H.; Duong, D. L.; Ta, Q. H., Yao, F.; Vu, Q. A.; Jeong, H. Y.; Chae, S. H.; Lee, Y. H. Nondestructive Characterization of Graphene Defects. Adv. Funct. Mater., 2013, 23, 5183-5189.

(28) Ly, T. H.; Chiu, M.-H.; Li, M.-Y.; Zhao, J.; Perello, D. J.; Cichocka, M. O.; Oh, H. M.; Chae, S. H.; Jeong, H. Y.; Yao, F.; Li, L.-J.; Lee, Y. H. Observing Grain Boundaries in CVD-Grown Monolayer Transition Metal Dichalcogenides. ACS Nano 2014, 8, 1140111408.

(29) Ly, T. H.; Zhao, J.; Cichocka, M. O.; Li, L.-J., Lee, Y. H. Dynamical Observations on The Crack Tip Zone and Stress Corrosion of Two-Dimensional $\mathrm{MoS}_{2}$. Nat. Commun. 2017, 8, 14116

(30) Michail, A.; Delikoukos, N.; Parthenios, J.; Galiotis, C.; Papagelis, K. Optical Detection of Strain and Doping Inhomogeneities in Single Layer $\mathrm{MoS}_{2}$. Appl. Phys. Lett. 2016, 108, 173102.

(31) Xiao, S.; Xiao, P.; Zhang, X.; Yan, D.; Gu, X.; Qin, F.; Ni, Z.; Han, Z. J.; Ostrikov, K. Atomic-layer soft plasma etching of $\mathrm{MoS}_{2}$. Sci. Rept. 2016, 6, 19945.

(32) Lee, Y. H.; Yu, L.; Wang, H.; Ling, X.; Shi, Y.; Lin, C.-T.; Huang, J.-K.; Chang, M.-T., Chang, C.-S.; Dresselhaus, M.; Palacios, T.; Li, L.-J.; Kong, J. Synthesis and Transfer of Single-Layer Transition Metal Disulfides on Diverse Surfaces. Nano Lett. 2013, 13, 18521857.

(33) Ly, T. H.; Yun, S. J.; Thi, Q. H.; Zhao, J. Edge Delamination of Monolayer Transition Metal Dichalcogenides. ACS Nano, 2017, 11, 7534-7541.

(34) Conley, H. J.; Wang, B.; Ziegler, J. I.; Haglund, R. F.; Pantelides, S. T.; Bolotin, K. I. Bandgap Engineering of Strained Monolayer and Bilayer $\mathrm{MoS}_{2}$. Nano Lett. 2013, 13, 3626-3630. 
(35) Yang, L; Cui, X.; Zhang, J.; Wang, K.; Shen, M.; Zeng, S.; Dayeh, S. A.; Feng, L.; Xiang, B. Lattice Strain Effects on The Optical Properties of $\mathrm{MoS}_{2}$ Nanosheets. Sci. Rept. 2014, 4, 5649.

(36) He, K.; Poole, C.; Mak, K. F.; Shan, J. Experimental Demonstration of Continuous Electronic Structure Tuning via Strain in Atomically Thin $\mathrm{MoS}_{2}$. Nano Lett. 2013, 13, 2931-2936.

(37) Cheng, Y.; Zhu, Z.; Mi W.; Guo, Z.; Schwingenschlögl, U. Prediction of Twodimensional Diluted Magnetic Semiconductors: Doped Monolayer $\mathrm{MoS}_{2}$ Systems. Phys. Rev. B 2013, 87, 100401.

(38) Dolui, K.; Rungger, I.; Pemmaraju, C. D.; Sanvito, S. Possible Doping Strategies for $\mathrm{MoS}_{2}$ Monolayers: An ab initio Study. Phys. Rev. B 2013, 88, 075420.

(39) Smithe, K. K.; English, C. D.; Suryavanshi, S. V.; Pop, E. Intrinsic Electrical Transport and Performance Projections of Synthetic Monolayer $\mathrm{MoS}_{2}$ Devices. 2D Materials, 2016, 4, 011009.

(40) Najmaei, S.; Amani, M.; Chin, M. L.; Liu, Z.; Birdwell, A. G.; O’Regan, T. P.; Ajayan, P. M.; Dubey, M.; Luo, J. Electrical Transport Properties of Polycrystalline Monolayer Molybdenum Disulfide. ACS Nano, 2014, 8, 7930-7937.

(41) R. T. Brinkmann. Dissociation of Water Vapor and Evolution of Oxygen in the Terrestrial Atmosphere. Journal of Geophysical Research 1969, 74, 5355-5368.

(42) Kresse, G.; Furthmüller, J. Efficient Iterative Schemes for ab initio Total-energy Calculations using a Plane-wave Basis Set. Phys. Rev. B 1996, 54, 11169.

(43) Kresse, G.; Joubert. D. From Ultrasoft Pseudopotentials to the Projector Augmented-wave Method. Phys. Rev. B 1999, 59, 1758. 
TOC FIGURE

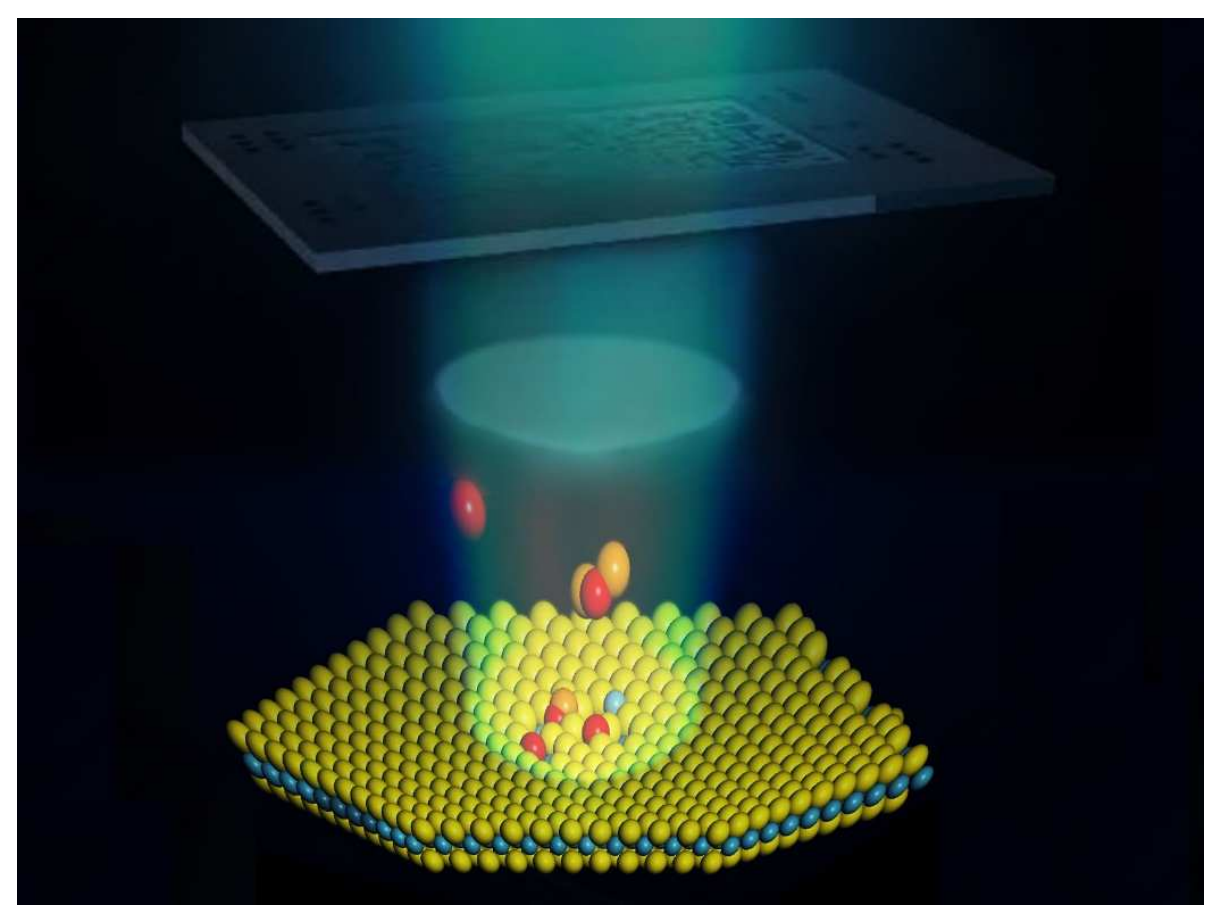

